\title{
Beyond morphological size distribution
}

\author{
Sébastien Lefèvre \\ University of Strasbourg-CNRS \\ Image Sciences, Computer Sciences, and Remote Sensing Laboratory (LSIIT) \\ Pôle API \\ Boulevard Brant \\ 67412 Illkirch, France \\ E-mail: lefevre@1siit.u-strasbg.fr
}

\begin{abstract}
In the field of digital image processing, the description of image content is one of the most crucial tasks. Indeed, it is a mandatory step for various applications, such as industrial vision, medical imaging, content-based image retrieval, etc. The description of the image content is achieved through the computation of some predefined features, which can be performed at different scales. Among global features that describe the content of the whole image, the gray level histogram focuses on the distribution of gray levels within the image, while morphological features (e.g., the pattern spectrum) measure the distribution of object sizes in the image. Despite their broad interest, such morphological size-distribution features are limited due to their monodimensional nature. Our goal is to review multidimensional extensions of these features able to deal with complementary information (such as shape, orientation, spectral, intensity, or spatial information). Moreover, we illustrate each multidimensional feature by an illustrative example that shows their relevance compared to the standard morphological size distribution. These features can be seen as relevant solutions when the standard monodimensional features fail to accurately represent the image content. @ 2009 SPIE and IS\&T. [DOI: 10.1117/1.3099707]
\end{abstract}

\section{Introduction}

In the field of digital image processing, the description of image content is one of the most crucial tasks. Indeed, it is a mandatory step for various applications, such as industrial vision, medical imaging, content-based image retrieval, etc. The description of the image content is achieved through the computation of some predefined features, which can be performed at different scales. The most elementary unit is the pixel itself, and it is possible to extract from a pixel its gray level (color or spectral signature in the case of multispectral images). If the neighborhood of the pixel is taken into account, its texture can also be measured through textural features. At an intermediary scale, one can segment the image into several regions and compute some features on each region. In this case, some shape or geometric information can be extracted, additionally, to average spectral and textural properties. Last, at a global level, some features can be computed to describe the whole image content. Some representative and complementary examples of global features are the gray level histogram and the pattern spectrum. While the gray level histogram focuses on the

Paper 08089R received Jun. 12, 2008; revised manuscript received Jan. 13, 2009; accepted for publication Feb. 4, 2009; published online Mar. 19, 2009.

1017-9909/2009/18(1)/013010/10/\$25.00 @ 2009 SPIE and IS\&T. distribution of gray levels within the image, the pattern spectrum measures the distribution of object sizes in the image.

Measuring the size distribution within an image is usually achieved by means of mathematical morphology, and more precisely through the use of morphological filters such as openings and closings. Granulometry, pattern spectrum, and covariance are some illustrative examples of such morphological-based features related to the size distribution and will be described in the next section. However, they are limited by their monodimensional nature (i.e., they measure only size information) and thus cannot gather complementary information. So our aim here is to review multidimensional extensions of these features able to deal with other kinds of information, such as shape, orientation, spectral, intensity, or spatial information. All these extensions will be presented next in dedicated sections with some illustrative examples to show their interest over standard sizedistribution features.

\section{Measuring the Distribution of Sizes with Mathematical Morphology}

\subsection{Mathematical Morphology}

Size distribution is usually measured by means of mathematical morphology, a theory introduced about 50 years ago by Georges Matheron and Jean Serra. Basically, it relies on the spatial analysis of images through a pattern called a structuring element (SE) and consists of a set of nonlinear operators that are applied on the images considering this SE. ${ }^{1}$ These operators are usually defined within the lattice theory framework. ${ }^{2}$ Let us note a digital image $f$ : $E \rightarrow T$, where $E$ is the discrete coordinate grid (usually $\mathbb{N}^{2}$ for a 2-D image), and $T$ is the set of possible image values. In the case of a binary image, $T=\{0,1\}$, where the objects and the background are respectively represented by values equal to 1 and 0 . In the case of a grayscale image, $T$ can be defined on $R$, but it is often defined rather on a subset of $\mathbb{Z}$, most commonly $[0,255]$. In the case of multidimensional images such as color images or multispectral or multimodal images, $T$ is defined on $\mathbb{R}^{n}$ or $\mathbb{Z}^{n}$, with $n$ the number of image channels. We will also denote by $p=(x, y)$ the pixel coordinates of an image. A complete lattice is defined from three elements: 
- A partially ordered set $(T, \geqslant)$, which could be the set inclusion order for binary images, the natural order of scalars for grayscale images, etc.

- An infimum or greatest lower bound $\wedge$, which is most often computed as the minimum operator. (This choice will also be made here for the sake of simplicity.)

- A supremum or least upper bound $V$, which is similarly most often computed as the maximum operator.

Once a complete lattice structure has been imposed on the image data, it is possible to apply morphological operators using a structuring pattern. This is called a structuring function (SF) or functional structuring element and noted $g$ when defined as a function on a subset of $T$, and called a structuring element (SE) and noted $b$ when defined as a set on $E$.

From these theoretical requirements, one can define the basic morphological operators known as erosion and dilation:

$\varepsilon_{b}(f)(p)=\bigwedge_{q \in b} f(p+q), \quad p \in E$,

$$
\delta_{b}(f)(p)=\underset{q \in b}{\vee} \underset{b}{f}(p+q), \quad p \in E,
$$

with $\breve{b}=\{-q \mid q \in b\}$ the reflexion of $b$. We will also use the notations $\varepsilon^{(n)}(f)=\varepsilon^{(1)}\left[\varepsilon^{(n-1)}(f)\right]$ and $\delta^{(n)}(f)=\delta^{(1)}\left[\delta^{(n-1)}(f)\right]$ to represent the successive applications of morphological operators. From these two operators, we can build most of the other morphological operators, in particular the opening and closing filters, which respectively remove bright and dark details:

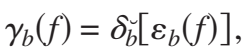

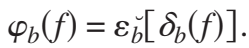

Beyond standard morphological filters also called structural filters, it is possible to build more advanced opening and closing filters to ensure a higher robustness to the choice of the SE $b$. Algebraic openings and closings assume a set $B=(b)_{i}$ of SE and are defined by:

$\gamma_{B}^{\alpha}(f)=\underset{b \in B}{\vee} \gamma_{b}(f)$

$\varphi_{B}^{\alpha}(f)=\underset{b \in B}{\wedge} \varphi_{b}(f)$,

and we will use the shortcut $\gamma_{\lambda}^{\alpha}=\gamma_{\lambda B}^{\alpha}$ with $\lambda B=(\lambda b)_{i}$ where $\lambda b$ denotes the SE $b$ scaled by a factor $\lambda$. Attribute opening is defined as

$\gamma_{\lambda}^{\chi}(f)=\underset{b}{\vee}\left\{\gamma_{b}(f) \mid b\right.$ is connected and $\left.\chi(b, \lambda)\right\}$

where $\chi(b, \lambda)$ denotes an attribute or criterion to be fulfilled. We can also cite the filters by reconstruction that rely on geodesic dilations and erosions; see Ref. 1 for a broad overview of morphological filters.

\subsection{Morphological Series and Size-Distribution Features}

Applying a morphological filter such as the opening with SE $b$ of increasing size $\lambda$ results in a series of successive filtered images with less and less details. Let us denote by $b_{\lambda}$ the SE $b$ of size $\lambda$, and write $\gamma_{\lambda}$ as a shortcut for $\gamma_{b_{\lambda}}$. We can then formulate the series $\Pi^{\gamma}(f)$ of successive openings $\gamma$ on the input image $f$ :

$\Pi^{\gamma}(f)=\left\{\Pi_{\lambda}^{\gamma}(f) \mid \Pi_{\lambda}^{\gamma}(f)=\gamma_{\lambda}(f)\right\}_{0 \leqslant \lambda \leqslant n}$

where $\gamma_{0}(f)=f$, and $n+1$ is the length of the series (including the original image). Instead of focusing on filtered images, one can also emphasize the details removed after each opening, thus building a differential series. Let us note as $\Delta^{\gamma}$ this series:

$\Delta^{\gamma}(f)=\left\{\Delta_{\lambda}^{\gamma}(f) \mid \Delta_{\lambda}^{\gamma}(f)=\Pi_{\lambda-1}^{\gamma}(f)-\Pi_{\lambda}^{\gamma}(f)\right\}_{0 \leqslant \lambda \leqslant n}$,

with the assumption that $\Delta_{0}^{\gamma}=0$. In this series, a pixel $p$ will appear (i.e., have a non-null value) in $\Delta_{\lambda}^{\gamma}(f)$ if it is removed by the morphological opening $\gamma_{\lambda}$ of size $\lambda$ [or in other words, if it was present in $\gamma_{\lambda-1}(f)$ but not anymore in $\left.\gamma_{\lambda}(f)\right]$.

From these two series $\Pi$ and $\Delta$, it is possible to compute the morphological features related to the distribution of object sizes within an image. Among these features, granulometry is built by gathering the values of the series $\Pi^{\gamma}$ over all pixels $p$ of the filtered image $\gamma(f)$ through a Lebesgue measure-for instance, a volume or sum operation. In the particular case of binary images, the image volume can either be computed as the sum of pixel values or as the amount of white pixels (or 1-pixels). The size distribution is then defined by:

$\Omega^{\gamma}(f)=\left\{\Omega_{\lambda}^{\gamma}(f) \mid \Omega_{\lambda}^{\gamma}(f)=\sum_{p \in E} \Pi_{\lambda}^{\gamma}(f)(p)\right\}_{0 \leqslant \lambda \leqslant n}$.

We can observe that $\Omega^{\gamma}$ is monotonically decreasing due to the extensivity property of the opening, i.e., $\gamma(f) \leqslant f$. In order for this measure to be invariant to image size and to represent cumulative distribution functions, it is worth being normalized, thus resulting in the new definition:

$\Gamma^{\gamma}(f)=\left\{\Gamma_{\lambda}^{\gamma}(f) \mid \Gamma_{\lambda}^{\gamma}(f)=1-\frac{\Omega_{\lambda}^{\gamma}(f)}{\Omega_{0}^{\gamma}(f)}\right\}_{0 \leqslant \lambda \leqslant n}$.

Notice that antigranulometry (also called anti-size distribution) can similarly be measured using a series of morphological closings $\Pi^{\varphi}$.

Another very interesting morphological global feature is the pattern spectrum $\Phi$ introduced by Maragos, ${ }^{3}$ also called pecstrum. ${ }^{4}$ It can be seen as the morphological counterpart of the well-known histogram. Instead of measuring the distribution of intensities within an image, it aims at measuring the distribution of sizes (and to a lesser extent, of shapes). To do so, it gathers values of the differential series $\Delta$ over all pixels: 

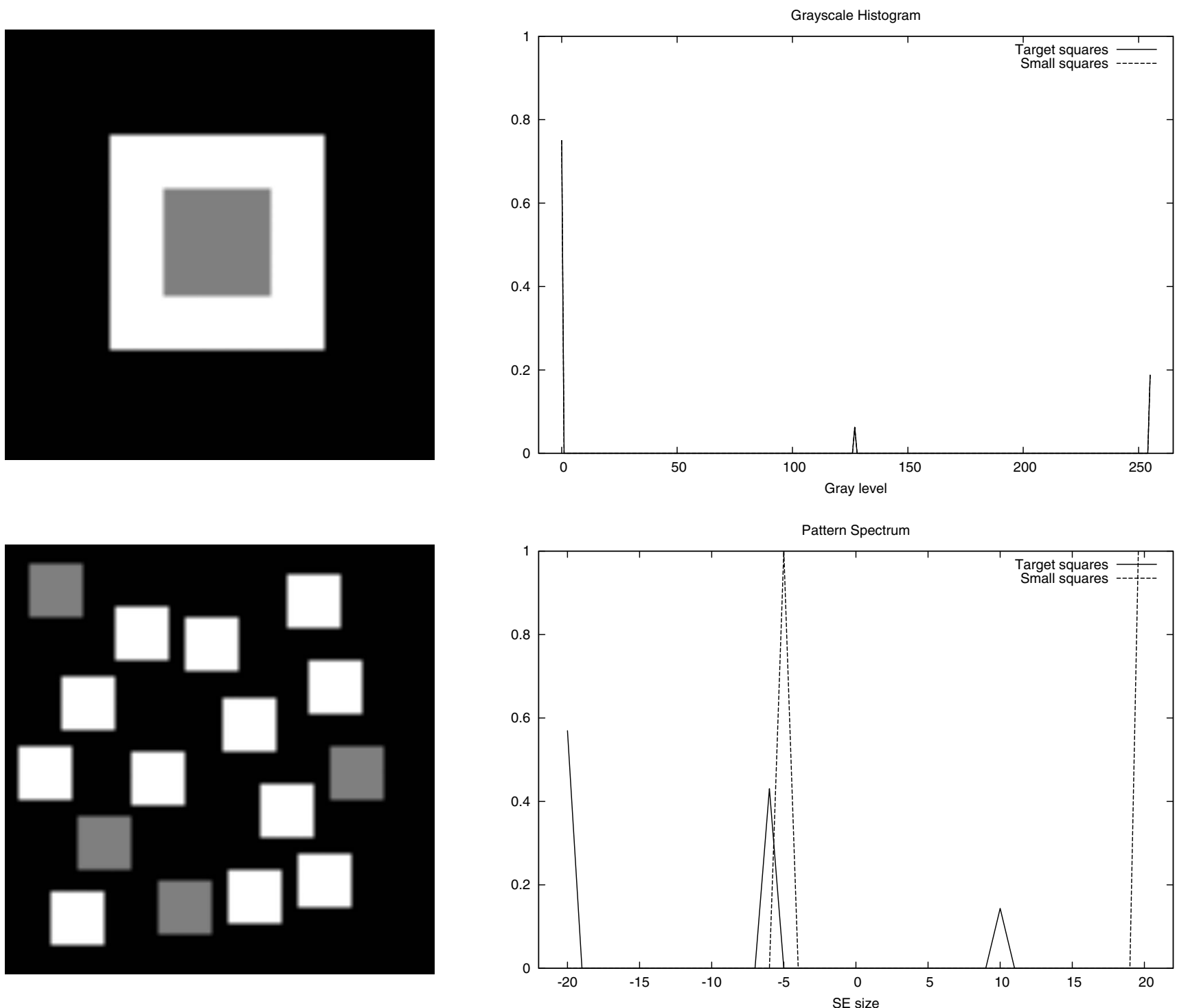

Fig. 1 Two input images (left) with similar histograms (top right) but different pattern spectra $\Lambda$ (bottom right).

$\Phi^{\gamma}(f)=\left\{\Phi_{\lambda}^{\gamma}(f) \mid \Phi_{\lambda}^{\gamma}(f)=\sum_{p \in E} \Delta_{\lambda}^{\gamma}(f)(p)\right\}_{0 \leqslant \lambda \leqslant n}$,

and the normalization ensures measures independent of the image size:

$\Lambda^{\gamma}(f)=\left\{\Lambda_{\lambda}^{\gamma}(f) \mid \Lambda_{\lambda}^{\gamma}(f)=\frac{\Phi_{\lambda}^{\gamma}(f)}{\Phi_{0}^{\gamma}(f)}\right\}_{0 \leqslant \lambda \leqslant n}$.

Moreover, Fig. 1 illustrates the relevance of the pattern spectrum in the case of an image with similar graylevel distribution. In this figure, negative and positive indices are respectively related to the use of opening and closing filters with increasing size $\lambda$.

In addition to the granulometry and the pattern spectrum relying on openings and closings, it is also possible to involve any morphological operator such as the erosion $\varepsilon$ to build a morphological feature able to describe the global image content. Thus, the covariance feature $K$ is defined as a morphological counterpart of the autocorrelation operator.
To compute this feature, the SE $b$ under consideration consists of a set of two points $p_{1}$ and $p_{2}$ and is defined by both a size $2 \lambda=\left\|\overrightarrow{p_{1} p_{2}}\right\|$ and an orientation $\vec{v}=\overrightarrow{p_{1} p_{2}} /\left\|\overrightarrow{p_{1} p_{2}}\right\|$ :

$K^{\vec{v}}(f)=\left\{K_{\lambda}^{\vec{v}}(f) \mid K_{\lambda}^{\vec{v}}(f)=\sum_{p \in E} \Pi_{\lambda, \vec{v}}^{\varepsilon}(f)(p)\right\}_{0 \leqslant \lambda \leqslant n}$,

where

$\varepsilon_{\lambda, \vec{v}}(f)(p)=f(p-\lambda \vec{v}) \wedge f(p+\lambda \vec{v})$.

Another definition of the covariance has been given by Serra ${ }^{5}$ where the autocorrelation function is used, thus resulting in the operator $\varepsilon^{\prime}$ defined by

$\varepsilon_{\lambda, \vec{v}}^{\prime}(f)(p)=f(p-\lambda \vec{v}) \cdot f(p+\lambda \vec{v})$,

where the intersection $\wedge$ is replaced by a product . operation. 
These features have been widely used to solve various problems such as classification, segmentation, and filtering. Moreover, size distribution presents some nice statistical properties that help to increase its interest since optimal features can be designed. These issues will not be addressed here, and the reader is referred to Ref. 6 (Chapter 8) for a more complete introduction to granulometries. However, even if these global features appear as particularly relevant alternatives to usual image features such as histograms, wavelets, or other textural features (just to mention a few), they still are limited to a single evolution curve and so cannot consider simultaneously several dimensions. More precisely, they deal only with the structural information extracted from morphological filters applied with growing SE sizes.

We review here some recent multidimensional extensions that allow us to build a 2-D series of morphological measures. These extensions help to gather complementary information (e.g., spatial, intensity, spectral, shape, etc.) in a single global morphological representation.

\section{Size-Shape Distribution}

In the standard size distribution, a unique parameter $\lambda$ was considered for measuring the size evolution, through the SE $b_{\lambda}$. This definition assumes a single size varying parameter $\lambda$ and prevents us from performing accurate measurements. Indeed, it is not adequate to elliptical or rectangular shapes, for instance, where the two independent axes should be taken into account. So several attempts have been made to build bivariate morphological series, thus allowing us to obtain size-shape measurements.

Lefèvre et ll. $^{7}$ consider structuring elements with two different size parameters $\alpha$ and $\beta$ that vary independently. More precisely, a way to define the 2-D series of SE $b_{\alpha, \beta}$ is given by $b_{\alpha, \beta}=\delta_{\check{\kappa}_{1}}^{(\alpha-1)}\left[\delta_{\check{\kappa}_{2}}^{(\beta-1)}(b)\right]=\delta_{\check{\kappa}_{2}}^{(\beta-1)}\left[\delta_{\check{\kappa}_{1}}^{(\alpha-1)}(b)\right]$, with $\kappa_{1}$ and $\kappa_{2}$ denoting the structuring elements used as growing factors in the two dimensions, and $b$ the initial SE. In the case of rectangular SE series, a relevant choice for $\kappa_{1}$ and $\kappa_{2}$ consists in 1-D SE such as horizontal and vertical lines, respectively (with a length proportional to the degree of coarseness desired), and an initial rectangular SE $b$.

The new II series built using the 2-D set of SE $b_{\alpha, \beta}$ is then computed as:

$$
\Pi^{\gamma}(f)=\left\{\Pi_{\alpha, \beta}^{\gamma}(f) \mid \Pi_{\alpha, \beta}^{\gamma}(f)=\gamma_{\alpha, \beta}(f)\right\}_{\substack{0 \leqslant \alpha \leqslant m \\ 0 \leqslant \beta \leqslant n}},
$$

where the application of $\gamma$ on $f$ with SE $b_{\alpha, \beta}$ is noted as $\gamma_{\alpha, \beta}(f)$ and with the convention $\gamma_{0,0}(f)=f$. Similarly, the $\Delta$ series measures the differential in both size dimensions:

$$
\begin{aligned}
\Delta^{\gamma}(f)= & \left\{\Delta_{\alpha, \beta}^{\gamma}(f) \mid \Delta_{\alpha, \beta}^{\gamma}(f)=\frac{1}{2}\left[2 \Pi_{\alpha-1, \beta-1}^{\gamma}(f)-\Pi_{\alpha-1, \beta}^{\gamma}\right.\right. \\
& \left.\left.-\Pi_{\alpha, \beta-1}^{\gamma}(f)\right]\right\}_{\substack{0 \leqslant \alpha \leqslant m \\
0 \leqslant \beta \leqslant n}},
\end{aligned}
$$

where $\Delta_{\alpha, 0}^{\gamma}=\Delta_{\alpha}^{\gamma}, \Delta_{0, \beta}^{\gamma}=\Delta_{\beta}^{\gamma}$, and $\Delta_{0,0}^{\gamma}=0$.

Figure 2 illustrates the potential interest of such 2-D features for sample images where standard pattern spectra are irrelevant.
A similar approach has been proposed by Ghosh and Chanda, ${ }^{8}$ who introduce conditional parametric morphological operators and who build a 2-D set of SE with increasing size, on both the horizontal and the vertical dimensions. From this set of SE, they finally compute the bivariate pattern spectrum for binary images. Bagdanov and Worring introduce the same feature under the term rectangular granulometry, ${ }^{9}$ while a slightly different definition has been given by Barnich et al. ${ }^{10}$ to limit the SE to the largest nonredundant rectangles within the analyzed object (in binary images). Moreover, a more general expression of $m$-parametric SE has been used in Ref. 11 to define multiparametric granulometries.

Batman et al. ${ }^{12,13}$ propose an alternative definition of this series using Euclidean series $\Pi^{\gamma^{\alpha}}(f)$ with the set of SE $B=\left\{-_{1},\left.\right|_{1}\right\}$, where - and $\mid$ denote, respectively, elementary horizontal and vertical SE. Moreover, they also introduce a univariate series by combining through the sum operations two series of SE $b_{\alpha}$ and $c_{\beta}$ built from initial SE $b$ and $c$ :

$$
\Pi^{\gamma}(f)=\left\{\Pi_{\alpha, \beta}^{\gamma}(f) \mid \Pi_{\alpha, \beta}^{\gamma}(f)=\gamma_{b_{\alpha}}(f)+\gamma_{c_{\beta}}(f)\right\}_{\substack{0 \leqslant \alpha \leqslant m \\ 0 \leqslant \beta \leqslant n}} .
$$

Urbach et al. ${ }^{14}$ also propose to combine size and shape information in a single 2-D granulometry. They rely on attribute filters ${ }^{15} \gamma^{\chi}$ and use a max-tree representation ${ }^{16}$ of the image for computational reasons. Their 2-D series can be defined as:

$\Pi^{\gamma^{\chi_{1}, \chi_{2}}}(f)=\left\{\Pi_{\alpha, \beta}^{\gamma}(f) \mid \Pi_{\alpha, \beta}^{\gamma}(f)=\gamma_{\alpha}^{\chi_{1}}(f) \wedge \gamma_{\beta}^{\chi_{2}}(f)\right\}_{\substack{0 \leqslant \alpha \leqslant m \\ 0 \leqslant \beta \leqslant n}}$,

where the two criteria $\chi_{1}$ and $\chi_{2}$ are respectively related to the area (i.e., defining size) and the ratio of the moment of inertia to the square of the area (i.e., defining shape).

\section{Size-Orientation Distribution}

Besides the size or shape of the SE, one can also vary its orientation. ${ }^{17}$ Naturally, this is relevant only with anisotropic structuring elements. Let us note as $b_{\lambda, \theta}$ an SE of size $\lambda$ and orientation $\theta$. This SE is built from a rotation of the initial SE $b_{\lambda}$ with an angle $\theta$, i.e., $\angle\left(b_{\lambda}, b_{\lambda, \theta}\right)=\theta$, with $\angle\left(b_{1}, b_{2}\right)$ the measured angle between orientations of $b_{1}$ and $b_{2}$.

Based on this principle, the morphological series is then defined as:

$$
\Pi^{\gamma}(f)=\left\{\Pi_{\lambda, \theta}^{\gamma}(f) \mid \Pi_{\lambda, \theta}^{\gamma}(f)=\gamma_{\lambda, \theta}(f)\right\}_{\substack{0 \leqslant \lambda \leqslant n \\ \theta_{0} \leqslant \theta \leqslant \theta_{m}}},
$$

where $\left\{\theta_{0}, \ldots, \theta_{m}\right\}$ represents the set (of cardinality $m+1$ ) of orientations considered, and $\gamma_{\lambda, \theta}$ is a shortcut for $\gamma b_{\lambda, \theta}$. Figure 3 illustrates the interest of such size-orientation features when the standard granulometry is useless.

Apart from the most simple angles (i.e., $\theta=k \pi / 4$ ), one has to tackle very carefully the problem of discretization for rotated SE. Accurate approximations can be obtained by periodic lines (see the work of Jones and Soille ${ }^{18}$ ) and require the use of several SEs to get an accurate discrete representation of a continuous segment. ${ }^{19}$ It is also possible to retain for each pixel at a given size only the maximum or minimum value from the results returned by the morphological filter with the various orientations. ${ }^{3}$ In this case, 

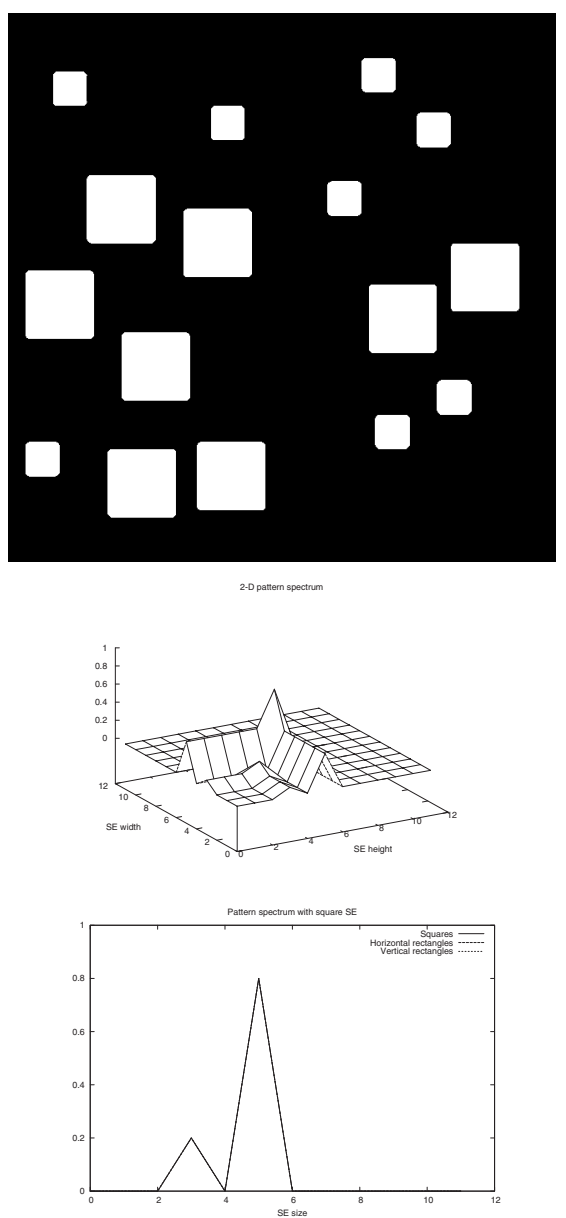

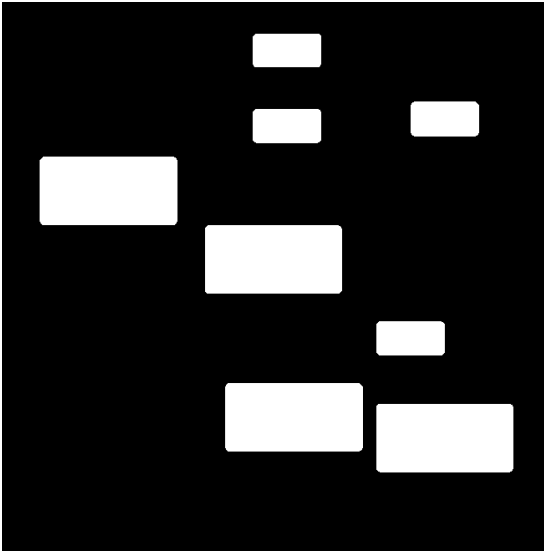

2-0 patter spectum
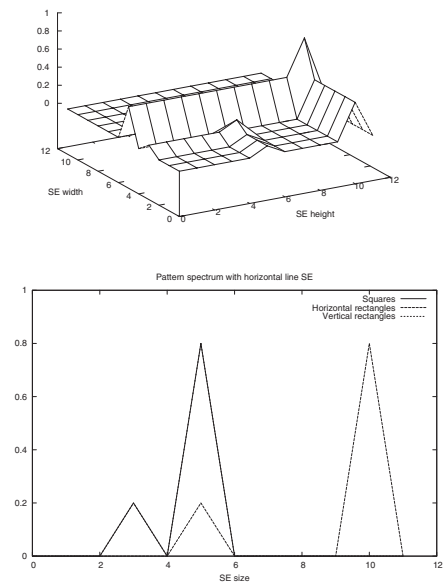

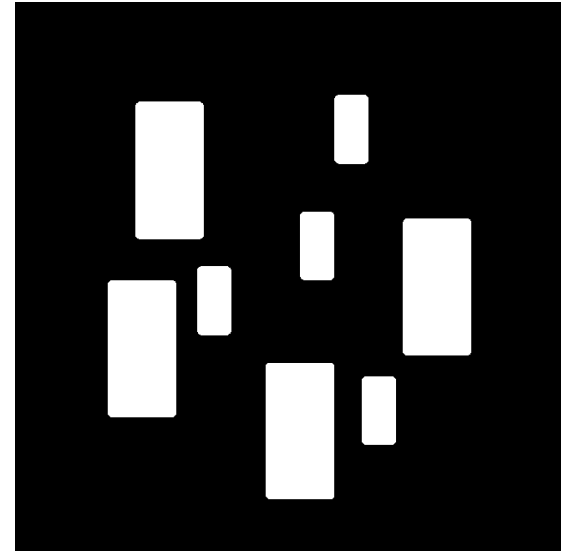

2-D pattem spectum
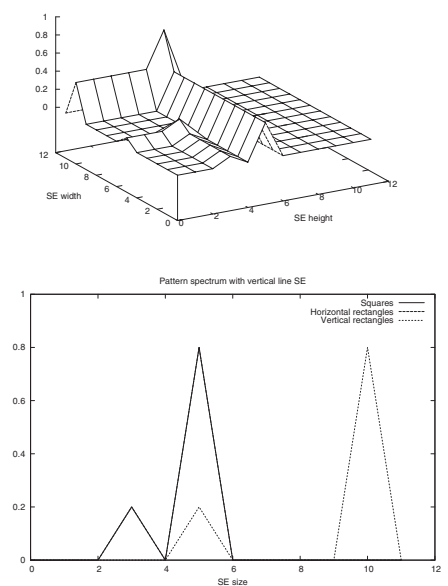

Fig. 2 Three input images (top) and their respective 2-D $\Delta$ features (middle). As a comparison, standard pattern spectra using square SE (bottom left), horizontal line SE (bottom center), and vertical line SE (bottom right) are also given.

however, the result is a 1-D series similar to the one that could be obtained by means of radial filters. ${ }^{1}$ Last, from these size-orientation measures, other features can be extracted such as orientation maps proposed by Soille and Talbot. $^{19}$

\section{Size-Spectral or Size-Color Distribution}

Since digital images contain very often spectral or color information, it is worth involving the spectral signature or color of each pixel in the computation of the morphological representation.

To do so, it is possible to first compute a morphological signature for each of the $k$ spectral components (or bands) and then to combine these $k$ signatures into a single one. With this two-step approach, the morphological series $\Pi$ can be expressed as:

$$
\Pi^{\gamma}(\mathbf{f})=\left\{\Pi_{\lambda, \omega}^{\gamma}(\mathbf{f}) \mid \Pi_{\lambda, \omega}^{\gamma}(\mathbf{f})=\gamma_{\omega}\left(f_{\omega}\right)\right\}_{\substack{1 \leqslant \omega \leqslant k \\ 0 \leqslant \lambda \leqslant n}},
$$

where $f_{\omega}$ is a grayscale image representing the $\omega$ 'th spectral component of the multispectral or colour image $\mathbf{f}=\left\{f_{\omega}\right\}_{1 \leqslant \omega \leqslant k}$. In this definition, morphological filters are applied independently on each image band, and thus the marginal strategy is used and the correlation among the different spectral channels is completely ignored. Moreover, this can result in new spectral signatures or colors in the filtered images.

To avoid these limitations, it is possible to rather consider a vectorial ordering when applying the morphological operators on the multispectral input image $\mathbf{f}^{20}$ The purpose of a vectorial ordering is to give a way to order vectors and thus to compute vectorial extrema by means of the two operators $\sup ^{v}$ and inf ${ }^{v}$. Assuming a given vectorial ordering, the fundamental dilation and erosion operators are written:

$\varepsilon_{b}^{v}(\mathbf{f})(p)=\inf _{q \in b}^{v} \mathbf{f}(p+q), \quad p \in E$,

$\delta_{b}^{v}(\mathbf{f})(p)=\sup _{q \in b}^{v} \mathbf{f}(p-q), \quad p \in E$,

and from these operators, it is possible to write all vectorial versions of the morphological operators described previously.

The new size-spectral morphological series is finally computed as: 

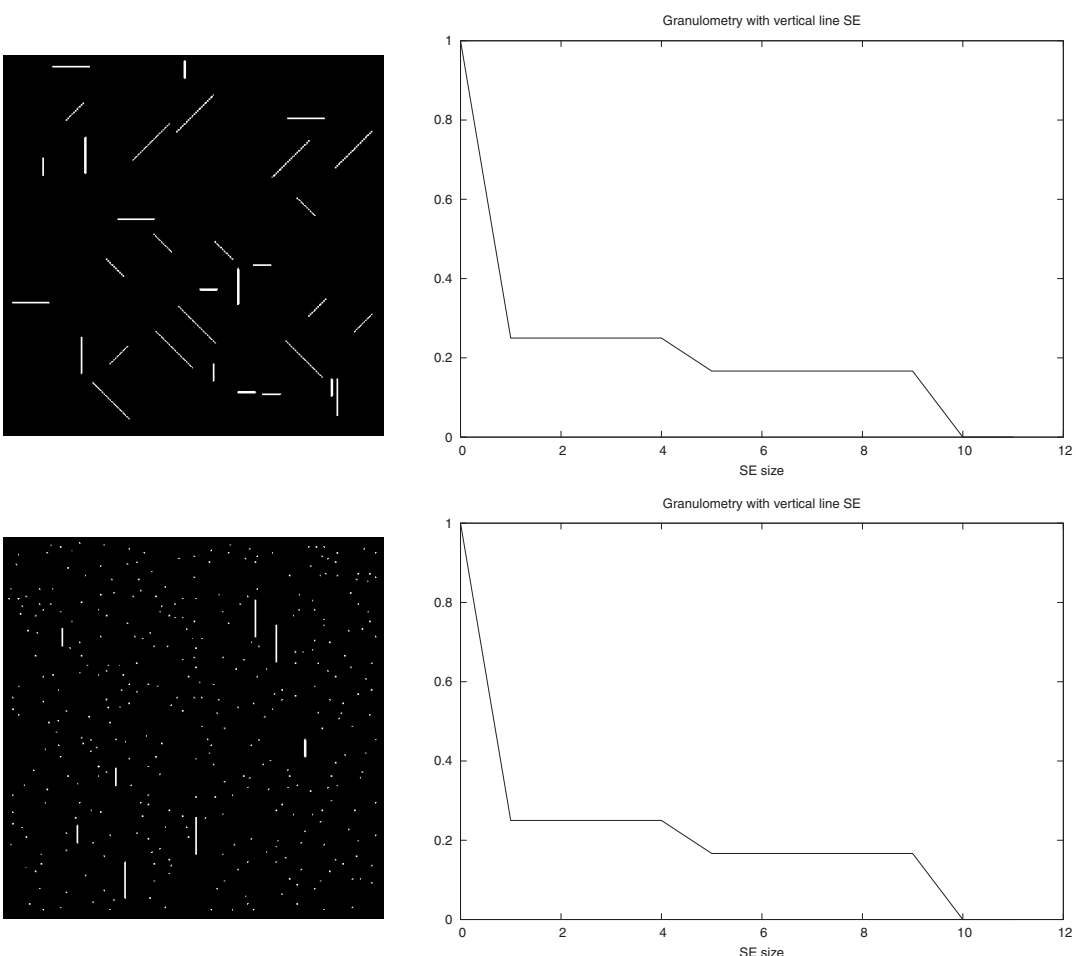
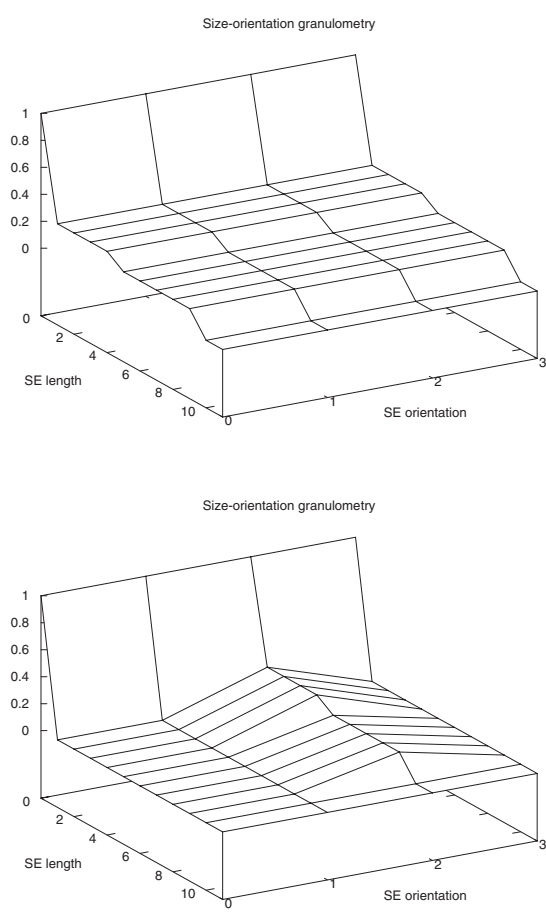

Fig. 3 Two input images (left), their respective (similar) granulometric curves with vertical SE (center), and their 2-D size-orientation granulometric curves (right) considering four angles.

$$
\Pi^{\gamma}(\mathbf{f})=\left\{\Pi_{\lambda, \omega}^{\gamma}(\mathbf{f}) \mid \Pi_{\lambda, \omega}^{\gamma}(\mathbf{f})=\left(\gamma_{\lambda}^{v}(\mathbf{f})\right)_{\omega}\right\}_{\substack{1 \leqslant \omega \leqslant k \\ 0 \leqslant \lambda \leqslant n}}
$$

where the values of $\gamma_{\lambda}^{v}(\mathbf{f})$ are relying on a given vectorial ordering. Let us note, however, that we have $\left[\gamma_{\lambda}^{v}(\mathbf{f})\right]_{\omega}$ $=\gamma_{\lambda}\left(f_{\omega}\right)$ in the specific case of a marginal ordering [thus, Eq. (22) is a particular case of the general formulation given in Eq. (25)]. A comparison of marginal and vectorial strategies is given in Fig. 4, considering a similar size distribution but a different spatial distribution in each color band.

For a comprehensive review of vectorial orderings and multivariate mathematical morphology, the reader can refer to the survey from Aptoula and Lefèvre. ${ }^{20}$ An example of color pattern spectrum can be found in Ref. 21, while a comparison between several vectorial orderings has also been proposed recently by Gimenez and Evans. ${ }^{22}$ In Ref. 23 , Nes and d'Ornellas consider color pattern spectra with linear SE of variable directions. (At each scale $\lambda$, the maximum pattern spectrum among the various orientations is selected.) In Ref. 24, Rivest deals with radar signals and propose adequate granulometry and power spectrum by introducing a vector ordering dedicated to complex data.

\section{Size-Intensity Distribution}

In grayscale images, the pixel intensity values are gathered with the sum operator. So the distribution of intensity values in the image is not taken into account with standard morphological features, which can be a real issue since intensity distribution (usually measured by a histogram) is a key feature to represent image content.
Computing the histogram on morphological scale-spaces has been proposed by Lefèvre ${ }^{25}$ to take into account both size and intensity distributions. To do so, let us use the Kronecker delta function:

$\delta_{i, j}=\left\{\begin{array}{ll}1 & \text { if } i=j \\ 0 & \text { if } i \neq j\end{array}\right.$,

and the histogram function $h_{f}: T \rightarrow \mathbb{Z}$ :

$h_{f}(\eta)=\sum_{p \in E} \delta_{\eta, f(p)}$

which measures the number of occurrences of each gray level $\eta$ in the image $f$. Alternatively, we can also use the normalized histogram function $h_{f}^{\prime}: T \rightarrow[0,1]$, where

$h_{f}^{\prime}(\eta)=\frac{h_{f}(\eta)}{|\operatorname{supp}(f)|}$,

with $|\operatorname{supp}(f)|$ the cardinality of the support of $f$, i.e., the number of pixels in $f$.

The formulation of the 2-D size-intensity morphological feature is then given by the following $\Pi$ series:

$\Pi^{\gamma}(f)=\left\{\Pi_{\lambda, \eta}^{\gamma}(f) \mid \Pi_{\lambda, \eta}^{\gamma}(f)=h_{\gamma_{\lambda}(f)}(\eta)\right\}_{\substack{\eta_{0} \leqslant \eta \leqslant \eta_{m} \\ 0 \leqslant \lambda \leqslant n}}$,

where $\left\{\eta_{0}, \ldots, \eta_{m}\right\}$ represents the different gray levels or bins in the histogram. 

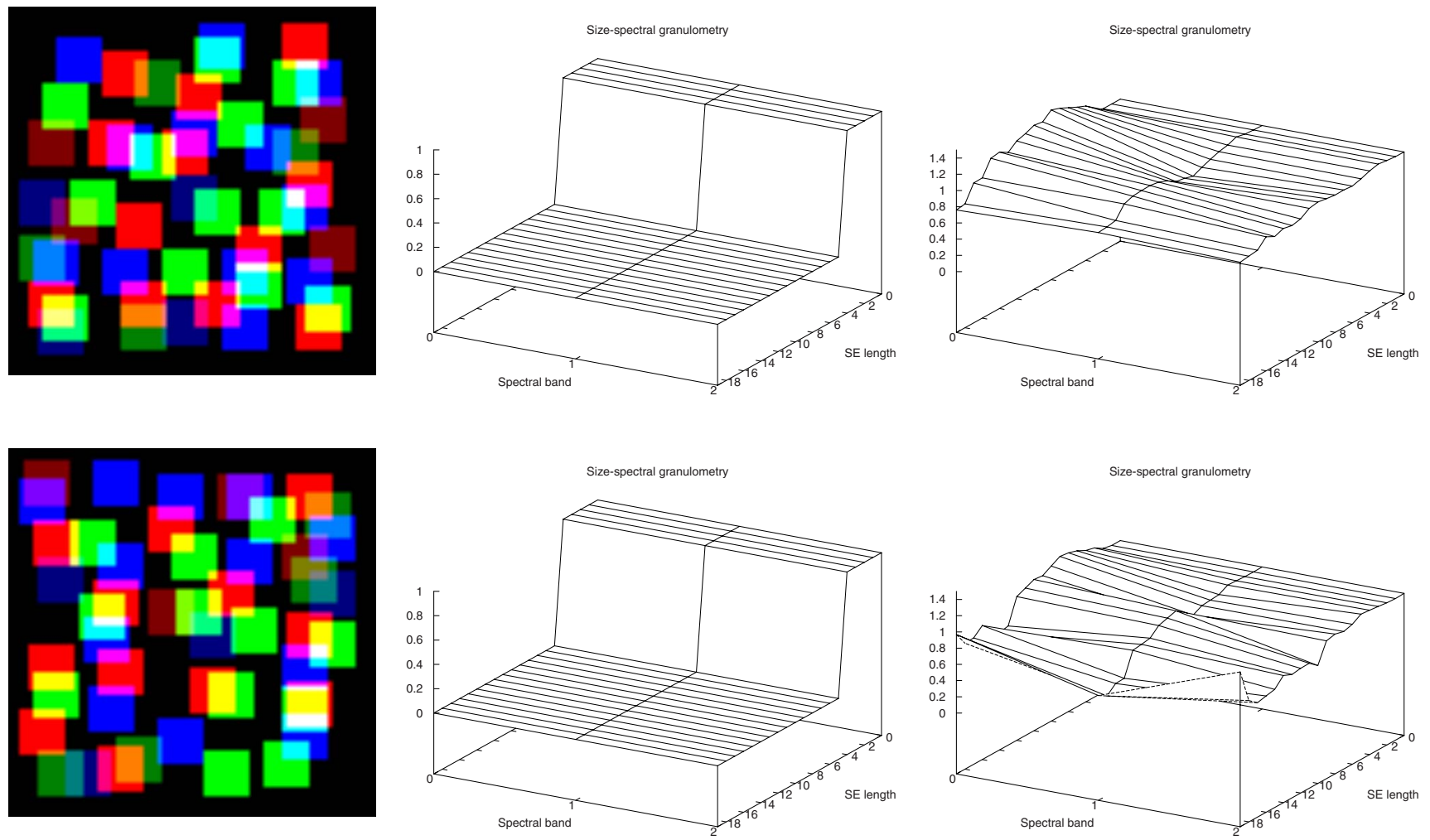

Fig. 4 Two input images (left), their respective granulometric curves computed with a marginal strategy (center), and with a vectorial strategy (right).
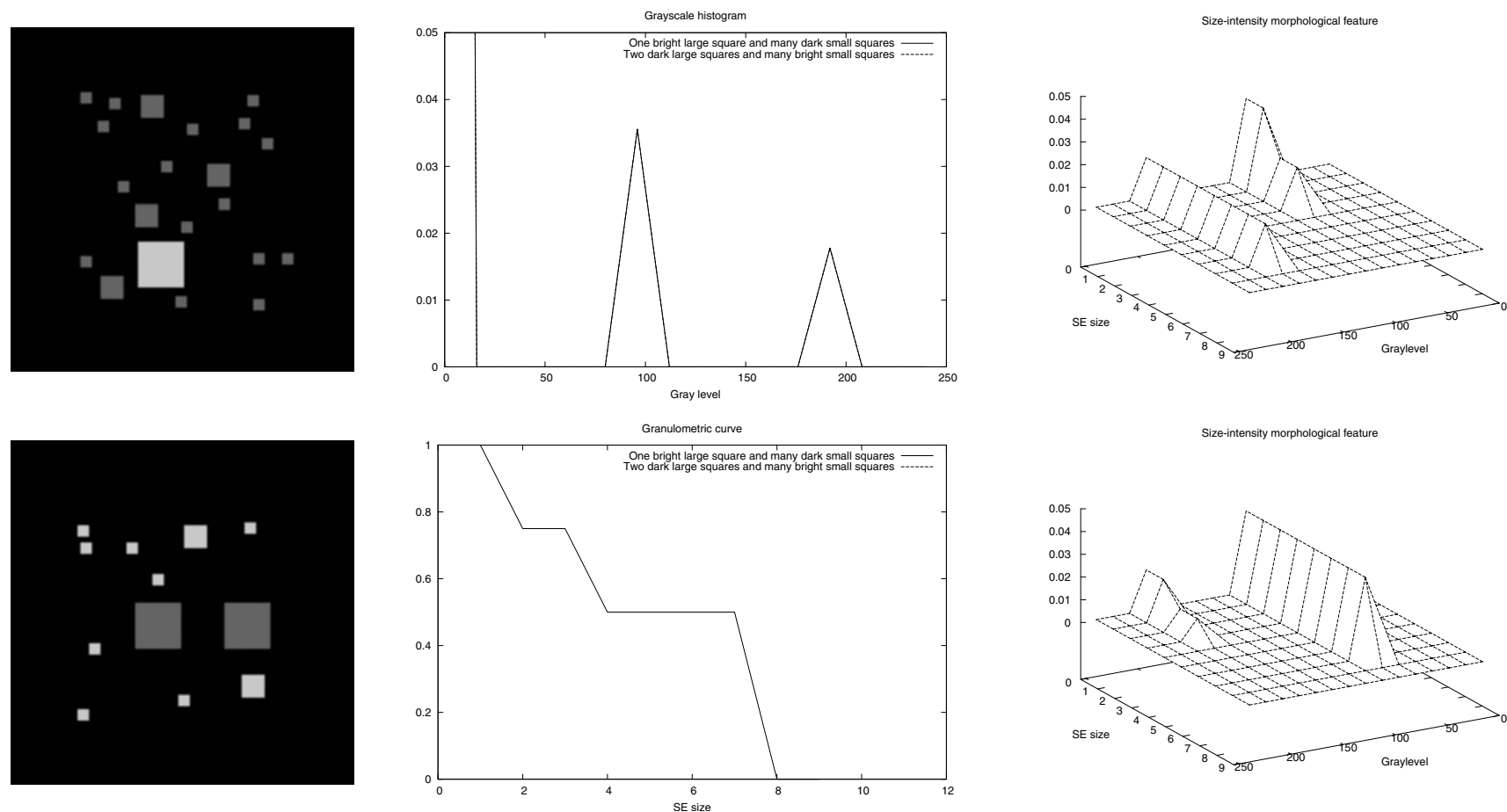

Fig. 5 Two input images (left) with similar histogram (top center) and granulometry (bottom center), but with different size-intensity morphological features (right). 
Figure 5 shows the relevance of size-intensity morphological features when both granulometry and histogram are irrelevant. For the sake of clarity, graylevel 0 (i.e., black pixels) has been omitted in the plots.

The derivative counterpart of the size-intensity morphological feature defined in Eq. (29) can be given by the following $\Delta$ series:

$\Delta^{\gamma}(f)=\left\{\Delta_{\lambda, \eta}^{\gamma}(f) \mid \Delta_{\lambda, \eta}^{\gamma}(f)=h_{\gamma_{\lambda}(f)-\gamma_{\lambda-1}(f)}(\eta)\right\}_{\substack{\eta_{0} \leqslant \eta \leqslant \eta_{m} \\ 0 \leqslant \lambda \leqslant n}}$.

This feature can be seen as a morphological alternative to the very effective multiresolution histograms computed from Gaussian linear scale-spaces. ${ }^{26}$

Spatial and intensity information can also be gathered by the use of structuring functions (SF) as proposed by Lotufo and Trettel. ${ }^{27}$ More precisely, let us define the SF $g_{\lambda, \eta}$ as a nonplanar cylinder of radius $\lambda$ and amplitude $\eta$. A sizeintensity feature is then built using various $\lambda$ and $\eta$ values:

$\Pi^{\gamma}(f)=\left\{\Pi_{\lambda, \eta}^{\gamma}(f) \mid \Pi_{\lambda, \eta}^{\gamma}(f)=\gamma_{\lambda, \eta}(f)\right\}_{\substack{\eta_{0} \leqslant \eta \leqslant \eta_{m} \\ 0 \leqslant \lambda \leqslant n}}$,

where $\gamma_{\lambda, \eta}$ is here a shortcut for $\gamma_{g_{\lambda, \eta}}$. It has been noted in Ref. 27 that both the classic histogram and the pattern spectrum can be derived from this measure by considering, respectively, $\lambda=0$ (i.e., a single pixel) and $\eta=0$ (i.e., a flat, disk-shaped SE).

A similar feature called a granold has been proposed by Jones and Jackway ${ }^{28}$ by first decomposing the grayscale image into a stack of binary images and then computing the granulometry for each binary image (i.e. at each grayscale threshold), thus resulting in the following series:

$\Pi^{\gamma}(f)=\left\{\Pi_{\lambda, \eta}^{\gamma}(f) \mid \Pi_{\lambda, \eta}^{\gamma}(f)=\gamma_{\lambda}\left[T_{\eta}(f)\right]\right\}_{\substack{\eta_{0} \leqslant \eta \leqslant \eta_{m} \\ 0 \leqslant \lambda \leqslant n}}$,

where $T_{\eta}$ denotes the thresholding function:

$T_{\eta}(f)(p)=\left\{\begin{array}{ll}1 & \text { if } f(p) \geqslant \eta \\ 0 & \text { if } f(p)<\eta\end{array}\right.$.

Despite their different definitions, both Refs. 27 and 28 lead to similar measures.

\section{Size-Spatial Distribution}

All the previous features were considering the spatial information through the successive applications of morphological operators that rely on a spatial neighborhood. But they did not retain any information about the spatial distribution of the pixels at a given scale $\lambda$. A first attempt to deal with this problem was made by Wilkinson, ${ }^{29}$ who proposed to compute spatial moments on the filtered binary images, thus resulting in spatial pattern spectra:

$\Phi^{\gamma}(f)=\left\{\Phi_{\lambda}^{\gamma}(f) \mid \Phi_{\lambda}^{\gamma}(f)=m_{i j}\left[\Delta_{\lambda}^{\gamma}(f)\right]\right\}_{\substack{m_{i j} \\ 0 \leqslant \lambda \leqslant n}}$,

where $m_{i j}$ denotes the moment of order $(i, j)$, computed on an image $f$ as:

$m_{i j}(f)=\sum_{(x, y) \in E} x^{i} y^{j} f(x, y)$
This idea was later followed by Aptoula and Lefèvre, ${ }^{30}$ where a normalized spatial covariance involving normalized unscaled central moments $\mu_{i j}$ is proposed to ensure scale and translation invariance:

$K^{\vec{v}}(f)=\left\{K_{\lambda}^{\vec{v}} \mid K_{\lambda}^{\vec{v}}=\mu_{i j}\left[\Pi_{\lambda, \vec{v}}^{\varepsilon}(f)(p)\right] / \mu_{i j}(f)\right\} \underset{\substack{\mu_{i j} \\ 0 \leqslant \lambda \leqslant n}}{ }$,

with $\mu_{i j}$ defined by:

$$
\begin{aligned}
\mu_{i j}(f)= & \frac{\sum_{(x, y) \in E}(x-\bar{x})^{i}(y-\bar{y})^{j} f(x, y)}{\left(m_{00}(f)\right)^{\alpha}} \\
& \text { with } \alpha=\frac{i+j}{2}+1, \quad \forall i+j \geqslant 2,
\end{aligned}
$$

and $\bar{x}=m_{10}(f) / m_{00}(f), \bar{y}=m_{01}(f) / m_{00}(f)$.

Alternatively, Ayala and Domingo proposed spatial size distributions, ${ }^{31}$ where filtered images of the morphological series are replaced by their intersection with filtered translated images, intersection being computed in a linear way with a product rather than in a nonlinear way with a minimum. Thus, their feature can be obtained by comparing the linear covariances applied on both initial and filtered images, for all possible vectors in a set defined by $\kappa b$, with increasing $\kappa$ values:

$$
\begin{aligned}
& \Omega(f)=\left\{\Omega_{\lambda, \kappa} \mid \Omega_{\lambda, \kappa}=\frac{1}{\left[\sum_{p \in E} f(p)\right]^{2}} \sum_{q \in \kappa b} K_{1}^{\prime \vec{q}}(f)\right. \\
& \left.\left.-K_{1}^{\prime} \vec{q}\left[\Pi_{\lambda}(f)\right]\right\}\right\}_{\substack{0 \leqslant \kappa \leqslant k \\
0 \leqslant \lambda \leqslant n}},
\end{aligned}
$$

where $\vec{q}$ is a shortcut for the vector $\overrightarrow{o q}$, with $o$ the center or origin of the SE $b$, and $q$ any neighbor belonging to the SE. Here, we have used the notation $K^{\prime}$ to denote the autocorrelation function. The size-spatial distribution can last be computed as a 2-D differential measure, in a way similar to the computation of the $\Delta$ measure from the associated $\Pi$ one. Zingman et al. $^{32}$ propose the pattern density spectrum with a rather similar definition but relying on some concepts of fuzzy sets. (Actually, their density opening operator is similar to a rank-max opening ${ }^{1}$ ) Combined with the standard pattern spectrum, they obtain the 2-D size-density spectrum.

Last, Aptoula and Lefèvre ${ }^{33}$ consider a composite SE built from two different SE and introduce two parameters $\lambda$ and $\kappa$ to deal with both the size of the two SEs and the shift between them. Their new operator combines the filtering properties of the granulometry and the covariance, thus resulting in a series:

$$
\Pi^{\gamma, \vec{v}}(f)=\left\{\Pi_{\lambda, \kappa}^{\gamma, \vec{v}}(f) \mid \Pi_{\lambda, \kappa}^{\gamma, \vec{v}}(f)=\gamma_{\lambda, \kappa \vec{v}}(f)\right\}_{\substack{0 \leqslant \kappa \leqslant k \\ 0 \leqslant \lambda \leqslant n}},
$$

with $\gamma_{\lambda, \kappa \vec{v}}$ a shortcut for $\gamma_{b_{\lambda, \kappa \vec{v}}}$, and the composite SE being defined as $b_{\lambda, \kappa \vec{v}}=b_{\lambda} \cup\left(b_{\lambda}+\kappa \vec{v}\right)$, i.e., a pair of SEs $b$ of size $\lambda$ separated by a vector $\kappa \vec{v}$. The following normalized measure can then be computed from the previous series: 

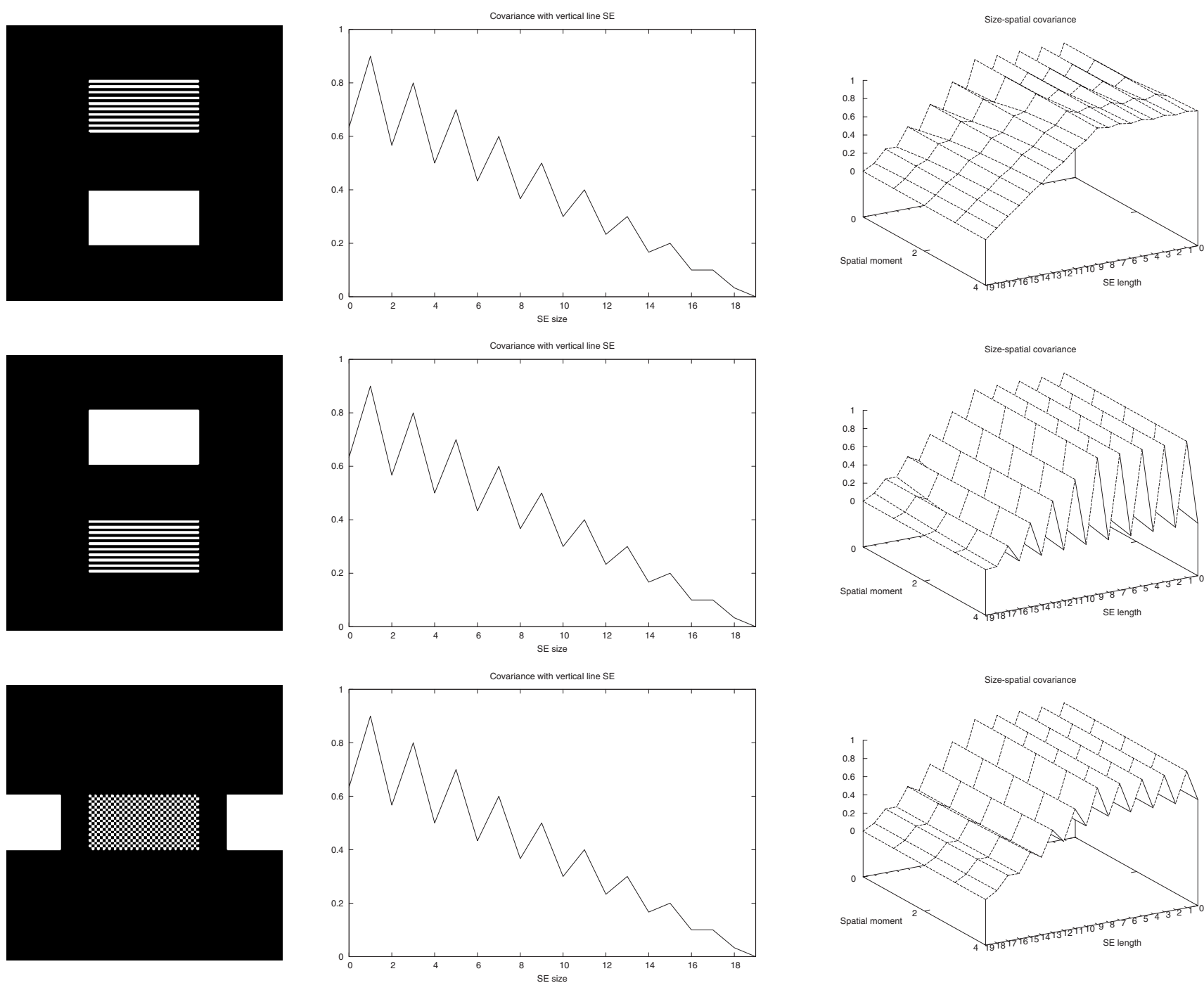

Fig. 6 Three input images (left), their respective covariance curve with vertical SE (center), and 2-D size-spatial granulometric curve (right) considering vertical spatial moments.

$$
\Gamma^{\gamma, \vec{v}}(f)=\left\{\Gamma_{\lambda, \kappa}^{\gamma, \vec{v}}(f) \mid \Gamma_{\lambda, \kappa}^{\gamma, \vec{v}}(f)=\frac{\sum_{p \in E} \Pi_{\lambda, \kappa}^{\gamma, \vec{v}}(f)(p)}{\sum_{p \in E} f(p)}\right\}_{\substack{0 \leqslant \kappa \leqslant k \\ 0 \leqslant \lambda \leqslant n}} .
$$

Figure 6 illustrates the relevance of such size-spatial features, considering the spatial covariance defined in Eq. (36) with vertical information taken into account.

\section{Conclusion}

Despite their broad interest in image description, the sizedistribution morphological features-namely, the granulometry, pattern spectrum, and covariance-are limited by their monodimensional nature. Thus, these features may not be relevant in many applications where other information has to be taken into account. In this paper, we review some extensions of the size distribution that combine size and other information in a single multidimensional feature, thus ensuring effective descriptions of the image content when size distribution is insufficient. The presented features deal respectively with shape, orientation, spectral, intensity, and spatial information.
In this paper, only 2-D features (i.e., features combining size with a single other characteristic) have been presented. However, one can design by induction any extension to the $n$-D case and thus easily build more expressive features.

We have given in this paper some illustrative examples to illustrate the relevance of the more than only sizedistribution features. We are now considering how to compare and evaluate these features in a real-life scenario, such as texture or object recognition. Moreover, future work will focus on implementation issues to avoid the brute force approach when computing these features and to ensure some efficient computation schemes.

\section{References}

1. P. Soille, Morphological Image Analysis Principles and Applications, Springer-Verlag, Berlin (2003).

2. C. Ronse, "Why mathematical morphology needs complete lattices," Signal Process. 21(2), 129-154 (1990).

3. P. Maragos, "Pattern spectrum and multiscale shape representation," IEEE Trans. Pattern Anal. Mach. Intell. 11(7), 701-716 (1989).

4. V. Anastassopoulos and A. N. Venetsanopoulos, "The classification properties of the pecstrum and its use for pattern identification," Circuits Syst. Signal Process. 10(3), 293-326 (1991). 
5. J. Serra, Image Analysis and Mathematical Morphology, Academic Press, London (1982).

6. E. R. Dougherty and R. A. Lotufo, Hands-on Morphological Image Processing, SPIE Press, Bellingham, WA (2003).

7. S. Lefèvre, J. Weber, and D. Sheeren, "Automatic building extraction in VHR images using advanced morphological operators," in IEEE/ ISPRS Joint Workshop on Remote Sensing and Data Fusion over Urban Areas, April 2007, Paris, France.

8. P. Ghosh and B. Chanda, "Bi-variate pattern spectrum," in Int. Symp. Computer Graphics, Image Processing, and Vision, pp. 476-483 (1998).

9. A. D. Bagdanov and M. Worring, "Granulometric analysis of document images," in IAPR Int. Conf. Pattern Recognition (ICPR), pp 468-471 (2002)

10. O. Barnich, S. Jodogne, and M. Van Droogenbroeck, "Robust analysis of silhouettes by morphological size distributions," in Int. Workshop on Advanced Concepts for Intelligent Vision Systems (ACIVS), Antwerp, Belgium, vol. 4179 of Lecture Notes in Computer Science, pp. 734-745, Springer-Verlag, Berlin (2006).

11. V. M. Gadre and R. K. Patney, "Multiparametric multiscale filtering, multiparametric granulometries, and the associated pattern spectral," in IEEE International Symposium on Circuits and Systems, pp. 15131516 (1992)

12. S. Batman and E. R. Dougherty, "Size distribution for multivariate morphological granulometries: texture classification and statistical properties," Opt. Eng. 36(5), 1518-1529 (1997).

13. S. Batman, E. R. Dougherty, and F. Sand, "Heterogeneous morphological granulometries," Pattern Recogn. 33, 1047-1057 (2000).

14. E. R. Urbach, J. B. T. M. Roerdink, and M. H. F. Wilkinson, "Connected shape-size pattern spectra for rotation and scale-invariant classification of gray-scale images," IEEE Trans. Pattern Anal. Mach. Intell. 29(2), 272-285 (2007).

15. E. J. Breen and R. Jones, "Attribute openings, thinnings, and granulometries," Comput. Vis. Image Underst. 64(3), 377-389 (1996)

16. P. Salembier, A. Oliveras, and L. Garrido, "Antiextensive connected operators for image and sequence processing," IEEE Trans. Image Process. 7(4), 555-570 (1998).

17. M. Werman and S. Peleg, "Min-max operators in texture analysis," IEEE Trans. Pattern Anal. Mach. Intell. 7(6), 730-733 (1985).

18. R. Jones and P. Soille, "Periodic lines: definitions, cascades, and application to granulometries," Pattern Recogn. Lett. 17, 1057-1063 (1996).

19. P. Soille and H. Talbot, "Directional morphological filtering," IEEE Trans. Pattern Anal. Mach. Intell. 23(11), 1313-1329 (2001).

20. E. Aptoula and S. Lefèvre, "A comparative study on multivariate mathematical morphology," Pattern Recogn. 40(11), 2914-2929 (2007).

21. A. Ledda and W. Philips, "Majority ordering and the morphological pattern spectrum," in Int. Workshop on Advanced Concepts for Intelligent Vision Systems (ACIVS), vol 3708 of Lecture Notes in Com puter Science, pp. 356-363, Springer-Verlag, Berlin (2005).
22. D. Gimenez and A. N. Evans, "An evaluation of area morphology scale-space for color images," Comput. Vis. Image Underst. 110 32-42 (2008).

23. N. Nes and M. C. d'Ornellas, "Color image texture indexing," in Int Conf. Visual Information and Information Systems, vol. 1614 of Lecture Notes in Computer Science, pp. 467-474, Springer-Verlag, Berlin (1999).

24. J. F. Rivest, "Granulometries and pattern spectra for radar signals," Signal Process. 86, 1094-1103 (2006).

25. S. Lefèvre, "Extending morphological signatures for visual pattern recognition," in IAPR Int. Workshop on Pattern Recognition in Information Systems (PRIS), pp. 79-88, INSPEC Press (2007).

26. E. Hadjidemetriou, M. D. Grossberg, and S. K. Nayar, "Multiresolution histograms and their use in recognition," IEEE Trans. Pattern Anal. Mach. Intell. 26(7), 831-847 (2004)

27. R. A. Lotufo and E. Trettel, "Integrating size information into intensity histogram," in Int. Symp. Mathematical Morphology (ISMM), pp. 281-288, Kluwer Academic Publishers, Boston (1996).

28. D. G. Jones and P. T. Jackway, "Granolds: a novel texture representation," Pattern Recogn. 33, 1033-1045 (2000).

29. M. H. F. Wilkinson, "Generalized pattern spectra sensitive to spatial information," in IAPR Int. Conf. Pattern Recognition (ICPR), vol. 1, pp. 21-24 (2002)

30. E. Aptoula and S. Lefèvre, "Spatial morphological covariance applied to texture classification," in Int. Workshop on Multimedia Content Representation, Classification, and Security (IWMRCS), vol. 4105 of Lecture Notes in Computer Science, pp. 522-529, SpringerVerlag, Berlin (2006)

31. G. Ayala and J. Domingo, "Spatial size distribution: applications to shape and texture analysis," IEEE Trans. Pattern Anal. Mach. Intell. 23(12), 1430-1442 (2001).

32. I. Zingman, R. Meir, and R. El-Yaniv, "Size-density spectra and their application to image classification," Pattern Recogn. 40, 3336-3348 (2007).

33. E. Aptoula and S. Lefèvre, "On morphological color texture characterization," in Int. Symp. Mathematical Morphology (ISMM), pp. 153-164, INPE, São José dos Campos, Brasil (2007).

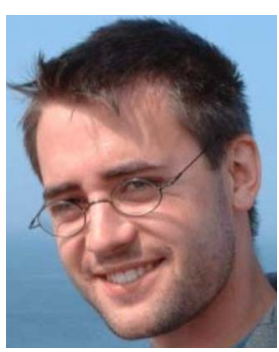

Sébastien Lefèvre received MSc and Eng degrees in computer engineering from the University of Technology of Compiègne in 1999 and a PhD degree in computer science from the University of Tours in 2002 $\mathrm{He}$ is currently an assistant professor in the Department of Computer Science and the LSIIT, CNRS-University of Strasbourg. His research interests are in image/video processing using mainly mathematical morphology with applications in remote sensing, astronomical and medical imaging, and content-based image/video retrieval. 